Claremont Colleges

Scholarship@ Claremont

All HMC Faculty Publications and Research

HMC Faculty Scholarship

5-22-1996

\title{
The Structure of Alkali Halide Dimers: A Critical Test of Ionic Models and New Ab Initio Results
}

T. Törring

Freie Universitat Berlin

S. Biermann

Freie Universitat Berlin

J. Hoeft

Freie Universitat Berlin

Richard J. Mawhorter

Pomona College

Robert J. Cave

Harvey Mudd College

See next page for additional authors

\section{Recommended Citation}

The structure of alkali halide dimers: A critical test of ionic models and new ab initio results. T. Torring, S. Biermann, J. Hoeft, R. Mawhorter, R. J. Cave, and C. Szemenyei, J. Chem. Phys. 104, 8032 (1996), DOI: 10.1063/1.471520

This Article is brought to you for free and open access by the HMC Faculty Scholarship at Scholarship @ Claremont. It has been accepted for inclusion in All HMC Faculty Publications and Research by an authorized administrator of Scholarship @ Claremont. For more information, please contact scholarship@cuc.claremont.edu. 
Authors

T. Törring, S. Biermann, J. Hoeft, Richard J. Mawhorter, Robert J. Cave, and C. Szemenyei 


\title{
The structure of alkali halide dimers: A critical test of ionic models and new ab initio results
}

\author{
T. Törring, ${ }^{\text {a) }}$ S. Biermann, and J. Hoeft \\ Institut für Experimentalphysik, Freie Universität Berlin, 14195 Berlin, Arnimallee 14, Germany \\ R. Mawhorter ${ }^{\text {a) }}$ \\ Pomona College, Department of Physics and Astronomy, 610 N. College Ave., Claremont, California 91711 \\ R. J. Cave ${ }^{\text {a) }}$ and C. Szemenyei \\ Department of Chemistry, Harvey Mudd College, Claremont, California 91711
}

(Received 12 October 1995; accepted 13 February 1996)

\begin{abstract}
In semiempirical ionic models a number of adjustable parameters have to be fitted to experimental data of either monomer molecules or crystals. This leads to strong correlations between these constants and prevents a unique test and a clear physical interpretation of the fit parameters. Moreover, it is not clear whether these constants remain unchanged when the model is applied to dimers or larger clusters. It is shown that these correlations can be substantially reduced when reliable information about dimers is available from experiments or $a b$ initio calculations. Starting with Dunham coefficients of the monomer potential determined from microwave measurements, we have calculated the monomer to dimer bond expansion and the bond angle without any additional adjustable parameter. Assuming that the overlap repulsion between nearest neighbors remains unchanged, the bond expansion is mainly determined by the simple Coulomb repulsion between equally charged ions and depends only very little on the effective ion polarizabilities. Deviation of the bond angle from $90^{\circ}$ sensitively tests the difference of effective polarizabilities of the two ions. A comparison with previously available data and new $a b$ initio MP2 results presented here for the heavy-atom containing dimers shows that bond angles can be modeled reasonably well with SeitzRuffa corrected Pauling polarizabilities while calculated bond expansions are much too long. This shows that changes of the overlap repulsion term must be considered for reliable predictions of the structure of dimers and larger clusters. (C) 1996 American Institute of Physics.
\end{abstract}

[S0021-9606(96)01519-3]

\section{INTRODUCTION}

Throughout more than six decades the apparent ionic character of the alkali halides has led to numerous attempts to understand both the crystal structure and the structure of diatomic molecules and clusters of intermediate size through a simple semiclassical model in which the attractive part of the potential is described in terms of classical electrostatics. As early as 1924 Born and Heisenberg ${ }^{1}$ tried to derive the binding energy and the bond distance of the diatomics from crystal data. No experimental data were available at that time to test the results. It is interesting to note, however, that already in their paper the important role of the mutual polarization of the ions which is suppressed by symmetry in the crystal has clearly been pointed out. Since then the potential energy in all these semiempirical models consists of the following terms:

$$
V=V_{\text {rep }}+V_{\text {Coul }}+V_{\text {pol }}\left(+V_{\text {vdW }}\right) .
$$

The first term is the quantum mechanical overlap repulsion between the ions, the second the mutual Coulomb attraction or repulsion, and the third term represents effects of mutual polarization. Later studies also added the van der Waals attraction term $V_{\mathrm{vdW}}$.

\footnotetext{
a) Authors to whom correspondence should be addressed.
}

When reliable experimental data for the diatomic alkali halides became available the interest in ionic models was renewed and many versions have been published during the past decades. ${ }^{2-26}$ A large variation in these models is possible because only the Coulomb term in Eq. (1) is straightforward, and all other terms can be modeled in many different ways resulting in a large number of possible combinations.

In the semiempirical theories considered here, the quantum mechanical overlap repulsion is modeled by an analytic function which usually has two adjustable parameters per ion pair. One example is the Born-Mayer potential ${ }^{27}$

$$
V_{\text {rep }}=A_{i k} \exp \left(-r_{i k} / \varrho_{i k}\right)
$$

which has been widely used, but a large number of other potentials have been explored. Recently, Kumar et al. ${ }^{24(\mathrm{~b})}$ have compared ten different forms of the repulsive potential. The two adjustable parameters are usually calculated from diatomic data by equating $r$ at the minimum of the potential with $r_{e}$, the experimental internuclear distance, and the first derivative of the potential with the force constant derived from the experimental vibrational frequency. Therefore, these two important quantities are usually input data of the model.

For the calculation of the polarization effects different sets of ion polarizabilities have been used, e.g., those of 
TABLE I. Parameters of the Born-Mayer potential from different models. ( $A$ in $10^{-9} \mathrm{erg}, \rho$ in $\AA$ ).

\begin{tabular}{|c|c|c|c|c|c|c|c|c|}
\hline \multirow[b]{2}{*}{ Monomer } & \multicolumn{2}{|c|}{ T-Rittner ${ }^{\mathrm{a}}$} & \multicolumn{2}{|c|}{ T-Rittner ${ }^{b}$} & \multicolumn{2}{|c|}{ DD model ${ }^{c}$} & \multicolumn{2}{|c|}{ Shell model ${ }^{\mathrm{d}}$} \\
\hline & $A$ & $\rho$ & $A$ & $\rho$ & $A$ & $\rho$ & $A$ & $\rho$ \\
\hline $\mathrm{LiF}$ & 1.2542 & 0.2725 & 2.24 & 0.252 & 0.5553 & 0.3052 & 0.3673 & 0.2990 \\
\hline $\mathrm{LiCl}$ & 1.5994 & 0.3310 & 4.28 & 0.341 & 0.5456 & 0.3800 & 0.5202 & 0.3420 \\
\hline $\mathrm{LiBr}$ & 1.6530 & 0.3499 & 5.74 & 0.314 & 0.6184 & 0.3961 & 0.6585 & 0.3530 \\
\hline LiI & 1.7888 & 0.3766 & 6.16 & 0.337 & 0.6677 & 0.4250 & 0.2615 & 0.4300 \\
\hline $\mathrm{NaF}$ & 2.1996 & 0.2821 & 3.85 & 0.259 & 1.3740 & 0.2974 & 0.4178 & 0.3300 \\
\hline $\mathrm{NaCl}$ & 2.4862 & 0.3367 & 5.28 & 0.306 & 1.2514 & 0.3628 & 2.0100 & 0.3170 \\
\hline $\mathrm{NaBr}$ & 2.4790 & 0.3553 & 6.29 & 0.313 & 1.3739 & 0.3784 & 1.6423 & 0.3400 \\
\hline $\mathrm{NaI}$ & 2.5563 & 0.3816 & 6.32 & 0.342 & 1.4544 & 0.4054 & 0.9794 & 0.3860 \\
\hline
\end{tabular}

${ }^{a}$ Reference 7(a). Calculated using free ion polarizabilities.

${ }^{\mathrm{b}}$ Reference 22. Calculated using Seitz-Ruffa corrected polarizabilities.

${ }^{\mathrm{c}}$ Reference $7(\mathrm{a})$.

${ }^{\mathrm{d}}$ Reference 11(a).

Pauling ${ }^{28}$ or of Coker. ${ }^{9}$ Free ion polarizabilities, however, do not give the correct molecular electric dipole moments when the induced moments in the ions are calculated by

$$
\mu_{i}=\alpha_{i} E_{i},
$$

where $E_{i}$ is the electric field at the $i$ th ion and $\alpha_{i}$ the free ion polarizability of this ion. This problem has been treated in many different ways which will be discussed later.

Unfortunately no clear cut decision can be made between the different versions of the model by a comparison with experimental monomer data. This is mainly due to the fact that the internuclear distance and the harmonic part of the potential have been used as input data to determine the adjustable parameters. Therefore, the main part of the potential is fixed to the experimental values and only the higher derivatives of the potential and the dissociation energy can be used to test the model. Here the differences between the models are not very large. Moreover, fitting to experimental data leads to strong correlations between $V_{\text {rep }}$ and the remaining parameters of the potential. This becomes clear when one looks at the constants $A$ and $\varrho$ of the Born-Mayer potential from different models shown in Table I. Obviously these "constants" are more model specific rather than ionpair specific as they should be and cannot be transferred from one model to the other.

This ambiguity of the models becomes more serious when applied to dimers or larger clusters. Berkowitz et al. ${ }^{14}$ have done calculations on neutral dimers, trimers, and tetramers and a number of ionic species using four different models. They found considerable differences among these in the predicted structures, dissociation energies and ionization potentials. On the other hand, additional information from the monomer to dimer bond expansion $\left(r_{e}^{\mathrm{dim}}-r_{e}^{\mathrm{mon}}\right)$ and bond angle $\Theta$ can be of great help in a critical comparison of the different models, especially if, as we will show, the ambiguities discussed above can be at least partly avoided.

The geometries of the alkali halide dimers have been studied experimentally with the electron diffraction method. ${ }^{29-33}$ For the lighter dimers the results are in good agreement with recent $a b$ initio calculations ${ }^{34-38}$ and experimental errors are small enough for a critical comparison with model calculations. The situation is less favorable for the heavier dimers. Comparison with results from ab initio calculations presented here indicates that including intramolecular multiple scattering effects ${ }^{39}$ is necessary in analyzing the electron diffraction data, and improves agreement with the theoretical results dramatically.

Additional and more precise information can be expected in the near future from IR and microwave work. Most recently we have succeeded in measuring the microwave spectrum of the mixed dimer $\mathrm{LiNaF}_{2}$, the first measurement of a rotational spectrum of an alkali halide dimer so far (to be published elsewhere). In addition to bond expansions and bond angles, electric dipole moments and hyperfine structure constants may become available from such experiments. These provide very important and sensitive tests to the applicability of ionic models and can be compared with recent $a b$ initio work on $\mathrm{LiNaF}_{2} \cdot{ }^{35}$ Quadrupole hyperfine structure can give an indication of possible changes in the ionic character of the bond.

Thermodynamic properties can also be derived from a knowledge of the structure and the vibrational frequencies, and a consolidation of the structural data will help constrain puzzling differences for the equilibrium concentrations of both mixed $^{40}$ and regular ${ }^{41-43}$ dimers. Finally there are a number of current studies of both mixed ${ }^{44}$ and regular alkali halide clusters, of which dimers are the simplest. The ability to understand and predict the structure of alkali halide dimers is still both useful and interesting.

\section{APPLICATION OF DIMER DATA TO HELP TEST IONIC MODELS}

The ionic models can be easily applied to dimers. When the adjustable parameters from the monomers are retained the dimer structure can be calculated without additional assumptions. However, the ambiguities discussed earlier are then transferred to the dimer results and no definite conclusions can be drawn from a comparison with experimental data to find an answer to the following questions. (i) Can predictions of dimer data be improved by a better modeling of the repulsive part of the monomer potential? (ii) How can 


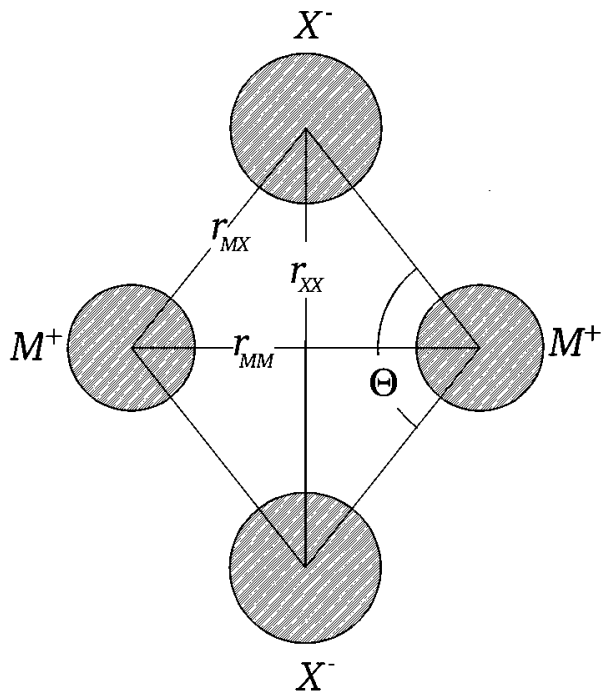

FIG. 1. Structure of the alkali halide dimers.

correlations between $V_{\text {rep }}$ and $V_{\text {pol }}$ be avoided in the model? (iii) What is the best way to calculate the polarization effects? (iv) Are there relevant deviations from 100\% ionic character? (v) Can the repulsive part of the monomer potential be transferred to the dimers without changes? The last question is of particular importance, because it is well known that repulsive parameters determined from crystals and monomer molecules differ considerably.

Problems connected with the first two questions can be completely avoided when dimer calculations start with experimental potential coefficients of the monomers rather than with a model potential. Accurate coefficients of the Dunham potential expansion ${ }^{45}$ have been derived for all alkali halides from microwave measurements. ${ }^{46}$ When these are used the critical overlap repulsion between ion pairs $\mathrm{M}^{+}-\mathrm{X}^{-}$is in full agreement with experimental monomer data and the rest of the dimer potential can be easily calculated in the framework of an ionic model:

$$
\begin{aligned}
V^{\mathrm{dim}}= & 4 V_{\mathrm{exp}}^{\mathrm{mon}}+e^{2}\left[\left(1 / r_{X X}\right)+\left(1 / r_{M M}\right)\right]+V_{\mathrm{pol}}^{\mathrm{dim}}-4 V_{\mathrm{pol}}^{\mathrm{mon}} \\
& +V_{\mathrm{rep}}(M, M)+V_{\mathrm{rep}}(X, X)+V_{\mathrm{vdW}}(M, M) \\
& +V_{\mathrm{vdW}}(X, X) .
\end{aligned}
$$

The second term on the right hand is the Coulomb repulsion between the two equally charged metal or halogen ions, respectively (see Fig. 1), the next two terms represent $\Delta V_{\text {pol }}$, the difference in the polarization energies of a dimer and four monomer bonds. In these terms the change of the polarizabilities plays an important role. The remaining terms give the additional overlap repulsion and van der Waals attraction between $\mathrm{M}^{+}-\mathrm{M}^{+}$and $\mathrm{X}^{-}-\mathrm{X}^{-}$ion pairs, respectively. The contribution of these terms is small compared to the others. Moreover they are of opposite sign and tend to cancel, so that they can be neglected in most cases. In this form there is no uncontrolled mixing of the influence of different terms by adjustable parameters, enabling a much more reliable test of the potential. Deviations of the ionic bond character $i_{c}$ from 1 can be included when the electron charge $e$ is replaced by $i_{c} \cdot e$ in the calculation of the electrostatic terms.

We will now examine the influence of the different terms on the geometry of the dimer in more detail. The additional Coulomb repulsion between $\mathrm{M}^{+}-\mathrm{M}^{+}$and $\mathrm{X}^{-}-\mathrm{X}^{-}$is symmetric, so the dimer will have a bond angle of $90^{\circ}$ if only this term is considered. This Coulomb repulsion is responsible for the major part of the bond expansion. A smaller contribution comes from the reduced polarization energy in the dimer. This reduction is not only due to the bond expansion caused by the additional Coulomb repulsion. The directions of induced dipole moments in the dimer don't coincide with the bond directions so that the attractive force along the bonds is reduced. Moreover we have a repulsive polarization potential between equally charged ions. While these effects can be calculated exactly by simple electrostatics the change of the ion polarizabilities can be treated in different ways and gives rise to some uncertainties. The calculations show, however, that the total $\Delta V_{\text {pol }}$ is less than $10-20 \%$ of the additional Coulomb repulsion potential. Therefore errors in the calculated bond expansion should be well below $10 \%$, even if the effective polarizabilities are not known very accurately. Larger deviations from reliable experimental values are a clear indication of other model errors, most likely that the monomer repulsive potential cannot be transferred to dimers without changes.

Deviation of the bond angle from $90^{\circ}$ is a sensitive measure of the asymmetry of the forces acting on the four ions. It should be noted that the bond angle is directly related to the difference of the polarizabilities of the ions while in monomers only the sum of the polarizabilities can be tested via dipole moments and bond energies. The influence of the van der Waals attraction and overlap repulsion terms is somewhat larger than on the bond expansion but will not exceed $2^{\circ}$, even for $(\mathrm{CsF})_{2}$ where it is largest.

We now have to consider the calculation of $\Delta V_{\mathrm{pol}}$. In Rittner's original paper ${ }^{2}$ the polarization energy in the monomers has been given (neglecting quadrupole and higher order terms) as

$$
\begin{aligned}
& V_{\mathrm{pol}}=-\frac{e}{r^{2}}\left(\mu_{M}+\mu_{X}\right)-2 \frac{\mu_{M} \mu_{X}}{r^{3}}+\frac{\mu_{M}^{2}}{2 \alpha_{M}}+\frac{\mu_{X}^{2}}{2 \alpha_{X}}, \\
& V_{\mathrm{pol}}=-\frac{e}{2 r^{2}}\left(\mu_{M}+\mu_{X}\right)-2 \frac{\mu_{M} \mu_{X}}{r^{3}},
\end{aligned}
$$

where the last two terms in Eq. (5a) represent the inner energy stored in the induced dipoles themselves. The induced moments $\mu_{M}$ and $\mu_{X}$ reduce the primary dipole moment $e \cdot r$ so that the net dipole moment of the molecule is given by $\mu=e \cdot r-\left(\mu_{M}+\mu_{X}\right)$. The induced moments are calculated by

$$
\mu_{M}=\alpha_{M}\left(e / r^{2}+2 \mu_{X} / r^{3}\right)
$$

and

$$
\mu_{X}=\alpha_{X}\left(e / r^{2}+2 \mu_{M} / r^{3}\right) .
$$


Brumer and Karplus ${ }^{7}$ have applied quantum mechanical exchange perturbation theory to the problem and have shown that in a consistent second-order perturbation theory the dipole-dipole interaction in the potential energy, Eq. (5), has to be omitted and only the first term occurs in Eqs. (6) and (7). This means that the contributions of the induced moments to the polarizing field are neglected in this so called "truncated" Rittner (T-Rittner) model. This second-order treatment is completely justified for the alkali halides and will be used in our model.

The problem of describing the induced dipole moments in the ions in accordance with experimental molecular dipole moments of the monomers has been tackled in many different ways. DeWijn ${ }^{47}$ assumed that the polarization of the halogen ion is partly quenched in the diatomic molecule due to increased overlap of the polarized electron shell with the electron shell of the metal. He postulated ad hoc that the polarizability of the halogen is reduced in the molecule to $2 / 3$ of its free ion value. Gowda et al. ${ }^{17}$ calculated the effective polarizabilities from the experimental dipole moments of the monomers using the Rittner and T-Rittner models. These effective polarizabilities were then used without changes for the dimer calculations. Brumer and Karplus ${ }^{7}$ found that in their second-order perturbation theory the repulsive term has to be written as

$$
V_{\text {rep }}=\left\{A_{M X}+\frac{B_{M X}}{2 r^{2}}\left(\alpha_{X}-\alpha_{M}\right)\right\} \exp \left(-r / \varrho_{M X}\right) .
$$

They noticed that this is equivalent to replacing the free ion polarizabilities by effective polarizabilities of the form $(e=1$ in Brumer's paper)

$$
\begin{aligned}
& \alpha_{X}^{\mathrm{eff}}=\alpha_{X}\left[1-r^{2} B_{M X} \exp \left(-r / \varrho_{M X}\right)\right], \\
& \alpha_{M}^{\mathrm{eff}}=\alpha_{M}\left[1+r^{2} B_{M X} \exp \left(-r / \varrho_{M X}\right)\right] .
\end{aligned}
$$

The ion pair specific adjustable parameter $B_{M X}$ was chosen such that the experimental dipole moments were exactly reproduced. The corrections to the free ion polarizabilities are considered in this model as due to overlap effects in the electron shells. The model is called the distortion dipole (DD) model.

A similar approach to the problem is that of Welch et al. ${ }^{11}$ In their shell model the repulsive term in the monomer is written as

$$
V_{\text {rep }}=A_{M X} \exp \left(-\frac{r+\mu_{M} / Q_{M}-\mu_{X} / Q_{X}}{\varrho_{M X}}\right) .
$$

The physical idea behind this model is to correct the distance of the ions for the shifts of the electron shells due to polarization. Two additional adjustable parameters in this model are the "effective shell charges" $Q_{X}$ and $Q_{M}$. These parameters are ion specific rather than ion-pair specific. They have been adjusted so that the model gives closest agreement with a great number of experimental data from alkali halide crystals and monomer molecules. It should be noted that the parameters $A_{M X}$ and $\varrho_{M X}$ have been fitted to crystal data in this model and that the free ion polarizabilities have likewise been fitted for best agreement with experimental data. They do not differ, however, very much from Pauling's free ion polarizabilities. Equation (11) can be written as

$$
\begin{aligned}
V_{\text {rep }} & =A_{M X} \exp \left[-\frac{e}{r^{2}}\left(\frac{\alpha_{M}}{\varrho_{M X} Q_{M}}-\frac{\alpha_{X}}{\varrho_{M X} Q_{X}}\right)\right] \exp \left(-r / \varrho_{M X}\right) \\
& \simeq\left\{A_{M X}+\frac{e A_{M X}}{r^{2} \varrho_{M X}}\left(\frac{\alpha_{X}}{Q_{X}}-\frac{\alpha_{M}}{Q_{M}}\right)\right\} \exp \left(-r / \varrho_{M X}\right) .
\end{aligned}
$$

Comparison with Eqs. (8), (9), and (10) shows that in this order of approximation the additional repulsive term of the shell model is equivalent to using effective polarizabilities in the calculation of the polarization energy which are given by

$$
\begin{aligned}
& \alpha_{X}^{\mathrm{eff}}=\alpha_{M}\left(1-\frac{2 r^{2} A_{M X}}{e Q_{X} \varrho_{M X}} \exp \left(-r / \varrho_{M X}\right)\right), \\
& \alpha_{M}^{\mathrm{eff}}=\alpha_{M}\left(1+\frac{2 r^{2} A_{M X}}{e Q_{M} \varrho_{M X}} \exp \left(-r / \varrho_{M X}\right)\right) .
\end{aligned}
$$

Obviously there is a very close resemblance to the DD model. The difference is mainly in the choice of the adjustable parameters.

A quite different approach is based on the Seitz-Ruffa (SR) energy level analysis. ${ }^{48,49}$ Here the free ion polarizabilities are changed by the additional Coulomb potential arising from the other ions. The polarizabilities are given by ${ }^{13}$

$$
\alpha_{\mathrm{eff}}=\frac{e^{2} \hbar^{2} n}{m\left(E_{f}+e \phi\right)^{2}},
$$

where $e$ and $m$ are the electron's charge and mass, respectively, $\hbar$ is Planck's constant, $n$ is the number of electrons in the ion, and $\phi$ is the electrostatic potential due to the other ions. $E_{f}$ is an energy parameter pertaining to the free ion. $E_{f}$ can be calculated from the free ion polarizability by

$$
\alpha=\frac{e^{2} \hbar^{2} n}{m E_{f}^{2}} .
$$

$\phi$ is negative at the position of the alkali ions and positive at the halogen ions, so that the polarizabilities of the metal ions are increased and those of the halogen ions are decreased in the molecule as in the other two models. No adjustable parameter is needed. Predictions of the monomer dipole moments are very near to the experimental results. In Table II experimental induced moments $\mu_{X}+\mu_{M}=e r_{e}-\mu_{\exp }$ are compared with calculations using Pauling's free ion polarizabilities and polarizabilities corrected with the SR and shell models, respectively. In most cases the SR results are much better than the predictions from the shell model. No comparison is possible with the DD model since the adjustable parameters $B_{M X}$ have been directly calculated from experimental dipole moments.

Both the SR and the DD models have a sound theoretical basis but give completely different arguments for the modification of the free ion polarizabilities. Since results of the SR theory are so good without using any adjustable parameters, it seems likely that it accounts for the major part of the corrections to the free ion polarizabilities, and that shell effects are much smaller than expected in Brumer's and 
TABLE II. Polarization dipole moments in the monomers (Debye).

\begin{tabular}{lcccc}
\hline \hline Monomer & Expt. & Pauling $^{\mathrm{a}}$ & Seitz-Ruffa $^{\mathrm{b}}$ & Shell model $^{\mathrm{c}}$ \\
\hline $\mathrm{LiF}$ & 1.23 & 2.10 & 1.31 & 0.43 \\
$\mathrm{LiCl}$ & 2.62 & 4.34 & 2.58 & 2.03 \\
$\mathrm{LiBr}$ & 3.20 & 4.89 & 3.25 & 2.20 \\
$\mathrm{LiI}$ & 4.06 & 5.98 & 4.11 & 2.99 \\
$\mathrm{NaF}$ & 1.13 & 1.58 & 1.19 & 0.93 \\
$\mathrm{NaCl}$ & 2.37 & 3.31 & 2.17 & 2.06 \\
$\mathrm{NaBr}$ & 2.92 & 3.80 & 2.71 & 2.19 \\
$\mathrm{NaI}$ & 3.81 & 4.76 & 3.45 & 2.78 \\
$\mathrm{KF}$ & 1.87 & 1.90 & 1.86 & -0.06 \\
$\mathrm{KCl}$ & 2.48 & 3.03 & 2.35 & 2.29 \\
$\mathrm{KBr}$ & 2.95 & 3.38 & 2.71 & 2.33 \\
$\mathrm{KI}$ & 3.59 & 4.10 & 3.24 & 2.62 \\
$\mathrm{RbF}$ & 2.39 & 2.27 & 2.37 & 2.82 \\
$\mathrm{RbCl}$ & 2.90 & 3.13 & 2.59 & 2.68 \\
$\mathrm{RbBr}$ & 3.28 & 3.42 & 2.87 & 2.78 \\
$\mathrm{RbI}$ & 3.78 & 4.04 & 3.33 & 2.92 \\
$\mathrm{CsF}$ & 3.41 & 3.02 & 3.40 & 3.28 \\
$\mathrm{CsCl}$ & 3.60 & 3.46 & 3.12 & 3.37 \\
$\mathrm{CsBr}$ & 3.96 & 3.66 & 3.30 & 3.25 \\
$\mathrm{CsI}$ & 4.23 & 4.16 & 3.62 & 3.35 \\
\hline \hline
\end{tabular}

${ }^{\mathrm{a}}$ Calculated with Paulings (Ref. 28) free ion polarizabilities.

${ }^{\mathrm{b}}$ Calculated with Seitz-Ruffa corrected polarizabilities (Ref. 13).

cFrom calculated bond lengths and dipole moments given in Ref. 11.

Welch's models. It would be interesting to repeat the calculation of the adjustable parameters $B_{M X}$ and $Q_{M}, Q_{X}$, respectively, using SR corrected instead of free ion polarizabilities. Bond angles of the dimers can help to clear up the problem, because they depend on the difference of the effective polarizabilities. An even more direct test will be possible when experimental dipole moments of mixed alkali halide dimers are available. We will use the SR correction in our calculations and compare the results with the DD and the shell-model of Welch et al.

Another nonempirical theory for the variation of ionic polarizabilities with site potential has been used to model the index of refraction in cubic crystals. ${ }^{50} \mathrm{~A}$ direct comparison with the SR results would require a high computational effort. However, from the numerical results given in Ref. 50 one can see that for most of the ions the free ion polarizabilities are near to those of Pauling. ${ }^{28}$ The $\mathrm{F}^{-}$ion is an important exception. Here the free ion polarizability is about $30 \%$ larger than the Pauling value. This large discrepancy is only partly canceled by a more rapid decrease of the polarizability with increasing site potential compared to the SR theory. When this larger polarizability is used in our calculations the results for monomer dipole moments and dimer structures are worsened.

The important influence of polarization effects on bond angles also shows up in nonempirical ionic models in which the mutual interaction between ions is calculated from an electron gas model. ${ }^{8}$ Such a model has been applied to some of the dimers by Trugman et al. ${ }^{10}$ neglecting deformations of the ions due to mutual polarization. This resulted in bond angles very near to $90^{\circ}$, confirming the arguments made in the aforementioned discussion. Recently it has been shown ${ }^{51}$ that an electron gas model which includes deformations of the ions gives improved results for crystal structures, especially in cases where anions occupy low symmetry positions. Much better bond angles can therefore be expected when this theory is applied to dimers.

\section{DETAILS OF THE CALCULATION}

\section{A. The ionic model}

Precise experimental data of the potential function of all alkali halides are available as coefficients $a_{0}$ to $a_{3}$ of the Dunham power series expansion ${ }^{45}$

$$
V(\xi)=a_{0} \xi^{2}\left(1+a_{1} \xi+a_{2} \xi^{2}+a_{3} \xi^{3}+\cdots\right)
$$

with

$$
\xi=\left(r-r_{e}\right) / r_{e} .
$$

$V(\xi)$ goes to minus infinity for large $r$ since $a_{3}$ is negative. This may lead to convergence problems in the numerical calculations of the potential minimum. Thakkar ${ }^{52}$ has proposed a potential expansion which is free from this shortcoming:

$$
V(r)=e_{0}(p) \lambda^{2}\left[1+e_{1}(p) \lambda+e_{2}(p) \lambda^{2}+e_{3}(p) \lambda^{3}+\cdots\right],
$$

where

$$
\lambda(r, p)=s(p)\left[1-\left(r_{e} / r\right)^{p}\right]
$$

with

$$
s(p)=\left\{\begin{array}{ll}
+1 & \text { for } p>0 \\
-1 & \text { for } p<0
\end{array} .\right.
$$

The coefficients of this series can be directly calculated from the Dunham coefficients: ${ }^{46}$

$$
p=-a_{1}-1 .
$$

For all alkali halides we have $p>1$ and therefore $s(p)=+1$. Then the coefficients $e_{i}(p)$ are

$$
\begin{aligned}
e_{0}(p)= & a_{0} / p^{2} \\
e_{1}(p)= & 0 \\
e_{2}(p)= & \left(a_{2}-1.5 p-7 p^{2} / 12-11 / 12\right) / p^{2}, \\
e_{3}(p)= & {\left[a_{3}+7 p / 4+p^{2}\left(2 e_{2}+7 / 6\right)+p^{3}\left(2 e_{2}+1 / 4\right)\right.} \\
& +5 / 6] / p^{3} .
\end{aligned}
$$

The Dunham potential is a special case of this generalized potential expansion.

The change of the polarization energy $\Delta V_{\mathrm{pol}}$ is given for homogeneous dimers by

$$
\begin{aligned}
\Delta V_{\mathrm{pol}}= & +2 e^{2}\left(\alpha_{M}^{\mathrm{mon}}+\alpha_{X}^{\mathrm{mon}}\right) / r_{M X}^{4}-e^{2} \alpha_{M}^{\operatorname{dim}}\left(r_{M M} / r_{M X}^{3}\right. \\
& \left.-1 / r_{M M}^{2}\right)^{2}-e^{2} \alpha_{X}^{\operatorname{dim}}\left(r_{X X} / r_{M X}^{3}-1 / r_{X X}^{2}\right)^{2} .
\end{aligned}
$$

First, the attractive polarization potential of the four monomer bonds is subtracted, and then the polarization potential of the dimer added. In both steps the adequate SR-corrected 
polarizabilities must be used [see Eqs. (15) and (16)]. For mixed dimers Eq. (22) is more complicated but straightforward.

Modeling of the overlap repulsion between equally charged ions is not very critical. We have applied the formalism proposed by Narayan and Ramaseshan ${ }^{53}$ where a Born-Mayer type potential with ion specific rather than ionpair specific constants is used. The repulsion between two ions $i$ and $k$ is written as

$$
V_{\text {rep }}\left(r, r_{i}, r_{k}\right)=A_{i} \exp \left(-r_{i} / \varrho_{i}\right)+A_{k} \exp \left(-r_{k} / \varrho_{k}\right)
$$

The "ionic radii", $r_{i}$ and $r_{k}$ are no fixed quantities but can be calculated from the distance $r$ between the ions and the ionic hardness parameters $\varrho_{i}$ and $\varrho_{k}$ :

$$
\begin{aligned}
& r_{i}=\left[r / \varrho_{k}-\ln \left(A_{k} \varrho_{i} / A_{i} \varrho_{k}\right)\right] /\left(1 / \varrho_{i}+1 / \varrho_{k}\right), \\
& r_{k}=\left[r / \varrho_{i}-\ln \left(A_{i} \varrho_{k} / A_{k} \varrho_{i}\right)\right] /\left(1 / \varrho_{i}+1 / \varrho_{k}\right) .
\end{aligned}
$$

For two identical ions we have simply $r_{i}=r_{k}=r / 2$. We have chosen this form of the repulsion potential because it can simply be applied also to mixed dimers. Tabulated data of $A_{i}$ and $\varrho_{i}$ can be found in Ref. 53(b).

Van der Waals attraction between two ions can be estimated by the Kirkwood-Muller formula (see Ref. 22 and references therein)

$$
V_{\mathrm{vdW}}=-\frac{6 m c^{2}}{r^{6} N} \frac{\chi_{i} \chi_{k}}{\left(\chi_{i} / \alpha_{i}+\chi_{k} / \alpha_{k}\right)},
$$

where $\chi_{i}$ and $\chi_{k}$ are the molar susceptibilities of the ions, $c$ is the velocity of light, $m$ is the electron mass, and $N$ is Avogadro's number. Values of the $\chi$ are listed in Ref. 24(b). We have calculated the van der Waals attraction using SRcorrected polarizabilities.

The potential energy of the dimer $V^{\mathrm{dim}}$ is a function of the bond length and angle. A computer program was written to find the minimum of this function. The program is based on an algorithm proposed by Nelder and Mead ${ }^{54}$ which can be applied to functions of an arbitrary number of variables. Hence, the program can be used for calculations of mixed dimers as well.

\section{B. $A b$ initio calculations}

In order to have a larger set of $a b$ initio dimer results with which to compare the ionic models, we have completed the series of dimer calculations begun recently in one of our labs. ${ }^{34,35}$ The calculations presented here are all second-order Moller-Plesset (MP2) results (thus they include electron correlation from a Hartree-Fock reference function). In our previous work we have found this to yield better absolute agreement with monomer bond lengths than Hartree-Fock results. However, it should also be noted that the bond length expansions from monomer to dimer were quite similar whether electron correlation was included or not. ${ }^{34,35}$ This is also the case for the systems presented here, but for brevity we only present MP2 results. In general the Hartree-Fock bond lengths tend to be longer (by 0.02-0.06 $\AA$ ). Monomer and dimer vibrational frequencies have been calculated at the Hartree-Fock level but are not included here.
The $\mathrm{Li}, \mathrm{Na}$, and $\mathrm{F}$ basis sets are those that were used in our previous study of the symmetrical dimers. ${ }^{34}$ In particular, the "polarized" basis sets based on the 6-31G set of Pople and co-workers were used. ${ }^{55}$ In the present study we used effective core potentials to describe $\mathrm{Cl}, \mathrm{Br}, \mathrm{I}, \mathrm{K} \mathrm{Rb}$, and $\mathrm{Cs}$. In addition, we have augmented the basis sets to include effects due to the ionic nature of the compounds and the core polarization effects. The individual effective core potentials and basis sets were the following.

$\mathrm{Cl}$. The effective core potential of Hay and $\mathrm{Wadt}^{56}$ was used, which replaces the $1 s, 2 s$, and $2 p$ electrons of $\mathrm{Cl}$. The basis set used was built on their $3 s, 3 p$ set (contracted to $2 s, 2 p$ ). Single $s$ and $p$ diffuse functions were added (exponents obtained based on an even tempered extension of the Hay and Wadt basis) and two $d$ functions (exponent $0.8,0.3$ ) were also included.

Br. The relativistic effective core potential of Hurley et $a l .{ }^{57}$ was used, which replaces the $1 s, 2 s, 2 p, 3 s, 3 p$, and $3 d$ electrons of $\mathrm{Br}$. The basis set used was built on their $3 s, 3 p$ set (contracted to $2 s, 2 p$ ). Single $s$ and $p$ diffuse functions were added (exponents obtained based on an even tempered extension of the Hurley et al. basis) and two $d$ functions (exponents 1.3, 0.3) were also included.

I. The relativistic effective core potential of LaJohn et $a l .{ }^{58}$ was used, which replaces the $1 s, 2 s, 2 p, 3 s, 3 p, 3 d$, $4 s, 4 p$, and $4 d$ electrons of I. The basis set used was built on their $3 s, 3 p$ set (contracted to $2 s, 2 p$ ). Single $s$ and $p$ diffuse functions were added (exponents obtained based on an even tempered extension of the LaJohn et al. basis) and two $d$ functions (exponents 1.3, 0.3) were also included.

$\mathrm{K}$. The relativistic effective core potential of Hurley et $a l .{ }^{57}$ was used, which replaces the $1 s, 2 s$, and $2 p$ electrons of K. In previous studies it has been found to be important for the description of the monomers and dimers to explicitly allow for polarization and correlation of the electrons in the $n-1$ shell of the alkali atoms. ${ }^{35}$ The basis set used was built on their $5 s, 4 p$ set (contracted to $4 s, 3 p$ ). Two $d$ functions (exponents $0.5,0.1$ ) were also included.

$\mathrm{Rb}$. The relativistic effective core potential of LaJohn et $a l .{ }^{58}$ was used, which replaces the $1 s, 2 s, 2 p, 3 s, 3 p$, and $3 d$ electrons of $\mathrm{Rb}$. The basis set used was built on their $5 s, 5 p$ set (contracted to $4 s, 3 p$ ). Two $d$ functions (exponents $0.5,0.1)$ were also included.

Cs. The relativistic effective core potential of Ross et $a l .{ }^{59}$ was used, which replaces the $1 s, 2 s, 2 p, 3 s, 3 p, 3 d$, $4 s, 4 p$, and $4 d$ electrons of Cs. The basis set used was built on their $5 s, 5 p, 4 d$ set (contracted to $4 s, 3 p, 2 d$ ). A $d$ function (exponent 0.5) was also included.

All calculations were performed using GAUSSIAN 92. ${ }^{60}$ The electrons correlated in the MP2 calculations were all valence electrons for the alkali and halide species, along with the electrons nominally in the $n-1 s$ and $p$ shells of the alkali atoms. The molecules were taken to be planar (preliminary Hartree-Fock vibrational frequencies indicated the molecules were planar) but $D_{2 h}$ symmetry was not enforced. Nevertheless the optimized geometries possessed essentially $D_{2 h}$ to within the numerical tolerances of the optimization procedure. The bond lengths reported are expected to be pre- 
TABLE III. New MP2 results for monomers and dimers.

\begin{tabular}{lcccccc}
\hline \hline System & $E_{\text {mon }}(h)$ & $r_{\text {mon }}(\AA)$ & $r_{e}(\AA)^{\mathrm{a}}$ & $\Delta r(\AA)$ & $\Theta_{\text {XMX }}$ & $\Delta E_{\text {bind }}(\mathrm{kcal} / \mathrm{mol})$ \\
\hline$(\mathrm{RbF})_{2}$ & -123.5644 & 2.334 & 2.2703 & 0.193 & 82.2 & 48.3 \\
$(\mathrm{CsF})_{2}$ & -119.6537 & 2.438 & 2.3453 & 0.227 & 78.4 & 43.4 \\
$(\mathrm{KCl})_{2}$ & -43.2179 & 2.690 & 2.6668 & 0.167 & 92.4 & 46.5 \\
$(\mathrm{RbCl})_{2}$ & -39.0811 & 2.799 & 2.7869 & 0.190 & 89.5 & 44.7 \\
$(\mathrm{CsCl})_{2}$ & -35.1704 & 2.937 & 2.9064 & 0.212 & 85.6 & 42.2 \\
$(\mathrm{LiBr})_{2}$ & -20.8184 & 2.200 & 2.1704 & 0.185 & 109.8 & 47.0 \\
$(\mathrm{NaBr})_{2}$ & -175.2403 & 2.534 & 2.5020 & 0.179 & 104.0 & 46.6 \\
$(\mathrm{KBr})_{2}$ & -41.3858 & 2.893 & 2.8208 & 0.187 & 95.6 & 43.2 \\
$(\mathrm{RbBr})_{2}$ & -37.2490 & 3.019 & 2.9447 & 0.201 & 92.7 & 41.4 \\
$(\mathrm{CsBr})_{2}$ & -33.3370 & 3.160 & 3.0722 & 0.222 & 89.0 & 39.3 \\
$(\mathrm{Lil})_{2}$ & -18.8486 & 2.414 & 2.3919 & 0.193 & 112.7 & 43.9 \\
$(\mathrm{Nal})_{2}$ & -173.2755 & 2.737 & 2.7115 & 0.182 & 107.5 & 44.3 \\
$(\mathrm{Kl})_{2}$ & -39.4195 & 3.124 & 3.0478 & 0.189 & 97.7 & 41.0 \\
$(\mathrm{Rbl})_{2}$ & -35.2834 & 3.267 & 3.1769 & 0.206 & 95.5 & 39.3 \\
$(\mathrm{CsI})_{2}$ & -31.3699 & 3.417 & 3.3152 & 0.226 & 91.5 & 37.5 \\
\hline \hline
\end{tabular}

${ }^{a}$ Experimental data from Ref. 7(a).

cise to within $0.001 \AA$, and for the dimers the bond expansions are taken relative to the average of the four $\mathrm{M}-\mathrm{X}$ bond lengths in the dimer. The bond angles are expected to be converged to better than $0.5^{\circ}$.

\section{RESULTS AND DISCUSSION}

The $a b$ initio results for the heavier atom dimers obtained in the present study are given in Table III. The data for $\mathrm{KCl}$ and $(\mathrm{KCl})_{2}$ provide useful points for comparison with our previous results. ${ }^{34}$ Here we have used effective core potentials for both $\mathrm{K}$ and $\mathrm{Cl}$, whereas we previously used all electron basis sets to describe these systems. The monomer bond length in Table III is in significantly better agreement with experiment $(2.667 \AA)$ than were our previous results $(2.739 \AA)$. We have found that this is most likely due to an "artificially small" $\mathrm{Cl}$ when using the ECP, and thus the good agreement is somewhat fortuitous. However, comparing the binding energies (present, $46.5 \mathrm{kcal} / \mathrm{mol}$; Ref. 34,

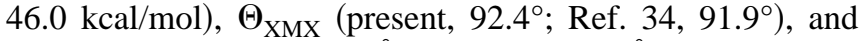
$\Delta r$ values (present, $0.167 \AA$; Ref. $34,0.167 \AA$ ) it is seen that the quantities of interest here are at most marginally altered by errors of the order of $0.05 \AA$ in the monomer bond lengths.

For the other halide containing species we find somewhat larger errors in the monomer bond lengths than are obtained for $\mathrm{Cl}$. The values are all longer than the experimental microwave values, with the largest errors occurring for CsI $(0.1 \AA)$. However, if one holds the halide atom fixed we obtain excellent changes in bond length with increasing size of the alkali atom. Good bond length changes are also obtained if the alkali atom is held constant in proceeding from $\mathrm{Br}$ to $\mathrm{I}$. As expected, given the $\mathrm{KCl}$ results discussed above, it is found that the $\mathrm{Cl}$ to $\mathrm{Br}$ change is somewhat larger than experiment.

The binding energies obtained here are in quite good agreement with experimental results (see Table IV). In fact, they are in significantly better agreement with experiment than one normally expects for $a b$ initio results at this level of theory. In our previous study on the symmetrical dimers we showed that basis set superposition effects (BSSE) had little to no effect on the dimerization energies obtained there at the self-consistent field (SCF) level. The basis sets used in the present study are of similar quality and we expect this to be the case for the systems examined here as well. We have examined BSSE at the MP2 level here, using a counterpoise correction based on treating the monomer at the MP2 level (at the monomer bond length) in the presence of the basis set for the second monomer, placed at the dimer bond distance from the monomer. For simplicity, a rectangular shape was assumed for the BSSE calculations. We have tested all $\mathrm{Br}$ containing species, and all $\mathrm{Rb}$ containing species, which allowed us to sample each atom type at least once. Corrections for BSSE at the MP2 level would reduce all $\mathrm{Br}$ containing bond strengths by approximately $3 \mathrm{kcal} / \mathrm{mole}$. For the other halide-containing dimers the bond strengths would be reduced by from 3.5 to $4.0 \mathrm{kcal} / \mathrm{mole}$. The relative uniformity of the corrections indicates that the trends found in Tables III and IV would not be altered significantly. In addition, more complete inclusion of correlation effects would likely increase the bond strengths again, partially offsetting the basis set superposition effects. We thus have presented the uncorrected values in Tables III and IV. It is likely that the relatively high accuracy stems from the fact that these are largely ionic systems, and that there is very little change in the ionic character of the alkali or halide as they are pulled apart to monomers. Indeed, we have found that in many cases Hartree-Fock binding energies are in quite good agreement with experiment.

Dissociation energies are often used as a test for ionic models. The dissociation energy relative to two monomers (dimerization energy) can be compared with experimental data

$$
D^{\mathrm{dim}}=D_{\text {ion }}^{\mathrm{dim}}-2 D_{\text {ion }}^{\text {mon }},
$$

where $D_{\text {ion }}^{\text {dim }}$ is the dissociation energy relative to free ions calculated from the model and $D_{\text {ion }}^{\text {mon }}$ is taken from experimental data. The Dunham or Thakkar expansions used in our model give the potential energy only relative to its value at 
TABLE IV. Dimerization energies ( $\mathrm{kcal} / \mathrm{mol})$.

\begin{tabular}{|c|c|c|c|c|c|c|}
\hline \multirow[b]{2}{*}{ Dimer } & \multirow[b]{2}{*}{ Expt. $^{a}$} & \multirow[b]{2}{*}{$A b$ initio $^{\mathrm{b}}$} & \multicolumn{2}{|c|}{ This model } & \multirow[b]{2}{*}{ Shell model ${ }^{\mathrm{d}}$} & \multirow[b]{2}{*}{ DD model ${ }^{\mathrm{a}}$} \\
\hline & & & $i_{c}=1^{\mathrm{c}}$ & $i_{c}=0.98$ & & \\
\hline$(\mathrm{LiF})_{2}$ & 63.6 & 63.4 & $52.6[51.1]$ & 63.8 & 53.0 & 60.6 \\
\hline$(\mathrm{LiCl})_{2}$ & 54.4 & 54.6 & $52.5[56.0]$ & 61.7 & 42.2 & 57.4 \\
\hline$(\mathrm{LiBr})_{2}$ & 49.3 & 47.0 & $56.1[61.3]$ & 64.8 & 41.7 & 53.1 \\
\hline$(\mathrm{LiI})_{2}$ & 44.4 & 43.9 & $57.9[64.8]$ & 66.0 & 26.7 & 52.5 \\
\hline$(\mathrm{NaF})_{2}$ & 59.3 & 59.8 & $53.4[51.5]$ & 62.7 & 43.4 & 57.8 \\
\hline$(\mathrm{NaCl})_{2}$ & 51.6 & 52.4 & $50.5[50.0]$ & 58.4 & 45.9 & 49.2 \\
\hline$(\mathrm{NaBr})_{2}$ & & 46.6 & $51.0[51.7]$ & 58.6 & 44.7 & 45.3 \\
\hline$(\mathrm{NaI})_{2}$ & 43.1 & 44.3 & $49.8[51.8]$ & 56.9 & 39.4 & 42.4 \\
\hline$(\mathrm{KF})_{2}$ & 49.3 & 51.1 & $46.8[45.9]$ & 55.3 & 41.0 & 47.9 \\
\hline$(\mathrm{KCl})_{2}$ & 45.4 & 46.5 & $44.5[43.5]$ & 51.6 & 41.0 & 41.4 \\
\hline$(\mathrm{KBr})_{2}$ & & 43.2 & $45.2[45.0]$ & 51.9 & 41.7 & 39.2 \\
\hline$(\mathrm{KI})_{2}$ & 39.9 & 41.0 & $42.5[42.9]$ & 48.8 & 39.4 & 36.4 \\
\hline$(\mathrm{RbF})_{2}$ & 45.7 & 48.3 & $42.7[41.4]$ & 51.0 & 36.2 & 47.6 \\
\hline$(\mathrm{RbCl})_{2}$ & 43.3 & 44.7 & $41.9[40.8]$ & 48.8 & 38.7 & 38.4 \\
\hline$(\mathrm{RbBr})_{2}$ & & 41.4 & $42.3[42.0]$ & 48.9 & 37.1 & \\
\hline$(\mathrm{RbI})_{2}$ & & 39.3 & $40.1[40.3]$ & 46.2 & 38.0 & \\
\hline$(\mathrm{CsF})_{2}$ & 41.3 & 43.4 & $40.2[36.8]$ & 48.4 & 42.4 & 50.1 \\
\hline$(\mathrm{CsCl})_{2}$ & & 42.2 & $44.7[43.3]$ & 51.4 & 37.8 & 33.4 \\
\hline$(\mathrm{CsBr})_{2}$ & & 39.3 & $46.5[46.0]$ & 52.9 & 37.8 & \\
\hline$(\mathrm{CsI})_{2}$ & & 37.5 & $43.5[43.5]$ & 49.4 & 36.6 & \\
\hline
\end{tabular}

Reference 7(b).

${ }^{\mathrm{b}}$ Reference 35 and this work (see Table III).

${ }^{c}$ Values in square brackets have been calculated with free ion polarizabilities.

${ }^{\mathrm{d}}$ Reference 11(a).

the monomer equilibrium distance $r_{e}$ and $D_{\text {ion }}^{\mathrm{dim}}$ cannot be calculated directly. So in our model the dimerization energy is given by

$$
D^{\mathrm{dim}}=2 D_{\text {ion }}^{\mathrm{mon}}-V^{\mathrm{dim}} .
$$

In both cases experimental errors of $D_{\text {ion }}^{\text {mon }}$ and $D^{\text {dim }}$ add up when the model is compared with experimental data. Brumer and Karplus ${ }^{7}$ estimate errors larger than $2.5 \mathrm{kcal} / \mathrm{mole}$ for most of the $D_{\mathrm{ion}}^{\text {mon }}$ with even greater errors for the fluorides. For the $D^{\mathrm{dim}}$ they assume errors of the order of $10 \%$ which is about $5 \mathrm{kcal} / \mathrm{mole}$. Within these large error bars results from all ionic models listed in Table IV are in reasonable agreement with experiment while the $a b$ initio results which are independent from the experimental $D_{\text {ion }}^{\text {mon }}$ are much better. It should be noted that only the DD model reproduces reasonably well the falling trend in the dimerization energies for each alkali going from the fluorides to the iodides. It is only this model that takes into account a change in the repulsive potential between monomers and dimers.

In addition, we have done the calculations with an assumed ionic character for both monomer and dimer of $98 \%$. The reduced repulsion between equally charged ions leads to an increase of $D^{\mathrm{dim}}$ by $6-10 \mathrm{kcal} / \mathrm{mole}$, considerably worsening agreement with experiment. The influence on the geometry of the dimer is small. Bond lengths are shortened by about $0.01 \AA$ and the bond angle is increased by $0.1^{\circ}-0.3^{\circ}$. So apparently small deviations from $100 \%$ ionic character can markedly change the dimerization energies but do not provide an improvement in the determination of dimer structures from ionic models.
Bond angles and expansions are listed in Tables $\mathrm{V}$ and VI. Data of recent $a b$ initio calculations (including those presented here) have been included for comparison. Since for the shell model monomer $r_{e}$ values are not fixed input data bond expansions have been given both with respect to calculated and experimental monomer values. To put comparison with experimental data on a more even footing, the experimental expansions given are with respect to thermally averaged monomer $r_{a}$ values based on the microwave $r_{e}$ values, vibrational frequencies, and anharmonicity parameters. From these the monomer $\left(r_{a}-r_{e}\right)$ difference is evaluated at the experimental temperature as discussed in Ref. 29. For the $\mathrm{X}-\mathrm{M}-\mathrm{X}$ bond angle the deviations from $90^{\circ}$ are listed since these are directly related to the asymmetry of the forces between equally charged ions and have to be explained by the models.

Unfortunately, due to monomer-dimer correlation the error bars of the experimental data ${ }^{29-33}$ from electron diffraction measurements are large. The quoted $2 \sigma$ uncertainties for the bond lengths are more than $10 \%$ of the bond expansion in all cases and go up to more than $50 \%$ for $(\mathrm{CsI})_{2}$ due to the small amount of dimer in the vapor. Again for the sake of consistency all experimental bond expansions given here come from analyses where all mean amplitudes of vibration were fixed to calculated values. ${ }^{29,30}$ Recently, $(\mathrm{CsCl})_{2}$ and $(\mathrm{KI})_{2}$ have been reanalyzed considering the effects of intramolecular multiple scattering. This has led to considerable changes of the geometry, outside the statistical error bars quoted in the original analysis. In the case of $(\mathrm{KI})_{2}$ this reanalysis has removed the obvious reversal of the bond 
TABLE V. Deviation of dimer bond angles $\Theta_{\mathrm{XMX}}$ from $90^{\circ}$.

\begin{tabular}{|c|c|c|c|c|c|c|c|}
\hline \multirow[b]{2}{*}{ Dimer } & \multicolumn{3}{|c|}{ Ionic models } & \multicolumn{3}{|c|}{$A b$ initio } & \multirow[b]{2}{*}{ Expt. } \\
\hline & This model $^{\mathrm{a}}$ & Shell model ${ }^{b}$ & DD model ${ }^{\mathrm{c}}$ & $\mathrm{MP}_{2}{ }^{\mathrm{d}}$ & $\mathrm{RHF}^{\mathrm{d}}$ & $\operatorname{LDA}^{\mathrm{e}}$ & \\
\hline$(\mathrm{LiF})_{2}$ & $+11.8[+19.2]$ & +7 & +12.5 & +11.7 & +9.5 & +10.0 & $+14.5(2.5)$ \\
\hline$(\mathrm{LiCl})_{2}$ & $+17.3[+28.5]$ & +8 & +20.7 & +18.0 & +17.5 & +17.9 & $+18.0(4.0)$ \\
\hline$(\mathrm{LiBr})_{2}$ & $+20.2[+29.8]$ & +21 & +22.1 & $+19.8^{\mathrm{f}}$ & & +20.4 & $+20.0(4.0)$ \\
\hline$(\mathrm{Lil})_{2}$ & $+22.5[+32.0]$ & +23 & +24.9 & $+22.7^{\mathrm{f}}$ & & & $+26.0(4.0)$ \\
\hline$(\mathrm{NaF})_{2}$ & $+5.7[+10.7]$ & -1 & +0.6 & +2.9 & +3.1 & +4.8 & $+4.7(0.7)$ \\
\hline$(\mathrm{NaCl})_{2}$ & $+12.2[+20.8]$ & +11 & +9.8 & +10.2 & +10.8 & +12.8 & $+11.4(2.4)$ \\
\hline$(\mathrm{NaBr})_{2}$ & $+15.0[+22.6]$ & +12 & +12.1 & $+14.0^{\mathrm{f}}$ & & +15.2 & $+11.6(1.8)$ \\
\hline$(\mathrm{Nal})_{2}$ & $+17.8[+25.6]$ & +15 & +15.6 & $+17.5^{\mathrm{f}}$ & & & $+12.5(4.6)$ \\
\hline$(\mathrm{KF})_{2}$ & $-4.2[+2.1]$ & -9 & -10.3 & -4.2 & -4.4 & -4.4 & $-5.3(1.6)$ \\
\hline$(\mathrm{KCl})_{2}$ & $+5.2[+13.4]$ & +3 & -1.7 & $\begin{array}{l}+1.9 \\
+24^{\mathrm{f}}\end{array}$ & $\begin{array}{l}+2.3 \\
+2.9^{\mathrm{f}}\end{array}$ & +3.7 & $+6.0(2.4)$ \\
\hline$(\mathrm{KBr})_{2}$ & $+8.3[+15.5]$ & +5 & -1.1 & $+5.6^{\mathrm{f}}$ & $+5.8^{\mathrm{f}}$ & +6.6 & $+5.8(1.4)$ \\
\hline$(\mathrm{Kl})_{2}$ & $+11.7[+18.9]$ & +8 & +4.9 & $+7.7^{\mathrm{f}}$ & $+7.8^{\mathrm{f}}$ & & $+8.6^{\mathrm{g}}(1.9)$ \\
\hline$(\mathrm{RbF})_{2}$ & $-8.9[-2.0]$ & -14 & -16.2 & $-7.8^{\mathrm{f}}$ & & & $-4.5(3.8)$ \\
\hline$(\mathrm{RbCl})_{2}$ & $+1.9[+10.3]$ & 0 & -7.3 & $-0.5^{\mathrm{f}}$ & $+0.2^{\mathrm{f}}$ & & $-1.8(2.0)$ \\
\hline$(\mathrm{RbBr})_{2}$ & $+5.3[+12.7]$ & +1 & & $+2.7^{\mathrm{f}}$ & $+3.2^{\mathrm{f}}$ & & $+4.7(1.8)$ \\
\hline$(\mathrm{Rbl})_{2}$ & $+9.0[+16.4]$ & +3 & & $+5.5^{\mathrm{f}}$ & $+5.7^{\mathrm{f}}$ & & $+7.2(4.2)$ \\
\hline$(\mathrm{CsF})_{2}$ & $-17.2[-8.7]$ & -18 & -23.2 & $-11.6^{\mathrm{f}}$ & & & $-7.5(3.8)$ \\
\hline$(\mathrm{CsCl})_{2}$ & $-3.5[+5.5]$ & -5 & -12.1 & $-4.4^{\mathrm{f}}$ & $-3.7^{\mathrm{f}}$ & & $-1.3^{\mathrm{g}}(3.6)$ \\
\hline$(\mathrm{CsBr})_{2}$ & $+0.5[+8.5]$ & -4 & & $-1.0^{\mathrm{f}}$ & $-0.9^{\mathrm{f}}$ & & $-5.0(1.8)$ \\
\hline$(\mathrm{CsI})_{2}$ & $+4.9[+12.8]$ & +1 & -7.3 & $+1.5^{\mathrm{f}}$ & $+1.5^{\mathrm{f}}$ & & $+6.5(8.2)$ \\
\hline
\end{tabular}

${ }^{a}$ Values in square brackets are calculated with free ion polarizabilities.

${ }^{\mathrm{b}}$ Reference 11.

${ }^{\mathrm{c}}$ Reference 7(b).

${ }^{\mathrm{d}}$ Reference 35 and this work (see Table III).

${ }^{\mathrm{e}}$ Reference 36.

${ }^{\mathrm{f}}$ Effective core calculations.

${ }^{\mathrm{g}}$ Analyzed including multiple scattering.

lengths of $(\mathrm{KI})_{2}$ and $(\mathrm{RbI})_{2}$. There is another such reversal for $(\mathrm{KBr})_{2}$ and $(\mathrm{RbBr})_{2}$. A thorough reanalysis of the experimental data using multiple scattering, gas phase vibrational frequencies, and the microwave monomer data would be highly desirable for a better test of ionic models and ab initio calculations. However, some conclusions can be drawn from the present available results, especially for the lighter dimers, where multiple scattering should have only a very small impact.

First we will consider the $\mathrm{X}-\mathrm{M}-\mathrm{X}$ bond angle $\Theta$. Obviously the calculations with fixed Pauling free ion polarizabilities give these angles systematically too high by $4^{\circ}-10^{\circ}$ compared to all other results. This underlines the experience from monomer dipole moments that the free ion polarizabilities have to be corrected in molecules. In all ionic models listed in Table $\mathrm{V}$ the polarizabilities of the halogens are decreased relative to the free ions while the metal polarizabilities are increased. This reduces the repulsive force along the $\mathrm{X}-\mathrm{X}$ axis and increases the force along the $\mathrm{M}-\mathrm{M}$ axis leading to a reduced $\mathrm{X}-\mathrm{M}-\mathrm{X}$ bond angle. The $\mathrm{DD}$ model is quite good for the Li compounds but becomes increasingly worse for the compounds of the heavier alkalis. This can be related to the fact that only one adjustable parameter $B_{M X}$ is responsible for the correction of both the metal and the halogen ion polarizabilities [see Eqs. (9) and (10)]. The polarizability of $\mathrm{Li}^{+}$is negligibly small, so for these compounds only the polarizabilities of the halides have to be corrected which can be done with one constant. When the metal polarizabilities become larger the difference of the polarizabilities is not modeled correctly and the bond angles of these compounds become too small.

In the shell model the two adjustable effective shell charges $Q_{M}$ and $Q_{X}$ give a greater flexibility for modeling the corrections to the polarizabilities [Eqs. (13) and (14)]. This model gives reasonable agreement with the present experimental bond angles except for the fluorides where the predicted angles are definitely too small.

The SR correction to the Pauling free ion polarizabilities needs no adjustable parameter but gives the best overall agreement with experiment including the fluorides, except for $(\mathrm{CsF})_{2}$ where the experimental data are in disagreement with all three models. This confirms the results from experimental monomer dipole moments that most, if not all, of the polarization correction can be explained by the SR theory. It should be noted that agreement with experimental and $a b$ initio values is best for those light dimers for which the monomer polarization corrections are also predicted with high accuracy (see Table II). Especially for the Li compounds, predictions of monomer dipole moments and dimer bond angles are excellent. For the bromides and iodides of the heavier alkalis the calculated polarization moments in the monomers are too small while the dimer bond angles tend to be too large. These discrepancies could be reduced by slightly increasing the effective metal polarizabilities in these compounds. As expected, the correlation of the bond angle with the repulsive potential is small. When in the calculation 
TABLE VI. Monomer to dimer bond expansion ( $)$.

\begin{tabular}{|c|c|c|c|c|c|c|c|}
\hline \multirow[b]{2}{*}{ Dimer } & \multicolumn{3}{|c|}{ Ionic models } & \multicolumn{3}{|c|}{$A b$ initio } & \multirow[b]{2}{*}{ Expt. } \\
\hline & This model & Shell model ${ }^{\mathrm{a}}$ & DD model ${ }^{\mathrm{b}}$ & $\mathrm{MP} 2^{\mathrm{c}}$ & $\mathrm{RHF}^{\mathrm{c}}$ & $\mathrm{LDA}^{\mathrm{d}}$ & \\
\hline$(\mathrm{LiF})_{2}$ & 0.247 & $0.24[0.12]$ & 0.184 & 0.144 & 0.152 & 0.148 & $0.151(20)$ \\
\hline$(\mathrm{LiCl})_{2}$ & 0.268 & $0.27[0.14]$ & 0.170 & 0.162 & 0.181 & 0.166 & $0.165(40)$ \\
\hline$(\mathrm{LiBr})_{2}$ & 0.277 & $0.27[0.19]$ & 0.193 & $0.185^{\mathrm{f}}$ & & 0.175 & $0.149(40)$ \\
\hline$(\mathrm{Lil})_{2}$ & 0.287 & $0.39[0.09]$ & 0.186 & $0.193^{\mathrm{f}}$ & & & $0.111(40)$ \\
\hline$(\mathrm{NaF})_{2}$ & 0.223 & $0.29[-0.03]$ & 0.159 & 0.152 & 0.142 & 0.131 & $0.128(10)$ \\
\hline$(\mathrm{NaCl})_{2}$ & 0.246 & $0.19[0.19]$ & 0.184 & 0.154 & 0.174 & 0.162 & 0.194 (34) \\
\hline$(\mathrm{NaBr})_{2}$ & 0.255 & $0.21[0.19]$ & 0.209 & $0.179^{f}$ & & 0.171 & 0.208 (34) \\
\hline$(\mathrm{Nal})_{2}$ & 0.265 & $0.24[0.16]$ & 0.227 & $0.182^{f}$ & & & $0.256(92)$ \\
\hline$(\mathrm{KF})_{2}$ & 0.241 & $0.29[0.08]$ & 0.182 & 0.176 & 0.178 & 0.188 & 0.148 \\
\hline \multirow[t]{2}{*}{$(\mathrm{KCl})_{2}$} & 0.257 & $0.20[0.17]$ & 0.201 & 0.168 & 0.191 & 0.186 & $0.249(54)$ \\
\hline & & & & $0.167^{\mathrm{f}}$ & $0.182^{\mathrm{f}}$ & & \\
\hline$(\mathrm{KBr})_{2}$ & 0.265 & $0.19[0.22]$ & 0.220 & $0.187^{f}$ & $0.203^{\mathrm{f}}$ & 0.189 & $0.346(22)$ \\
\hline$(\mathrm{Kl})_{2}$ & 0.272 & $0.20[0.23]$ & 0.243 & $0.189^{f}$ & $0.215^{\mathrm{f}}$ & & $0.323^{\mathrm{e}}(39)$ \\
\hline$(\mathrm{RbF})_{2}$ & 0.235 & $0.34[0.08]$ & 0.156 & $0.193^{\mathrm{f}}$ & - & & $0.152(76)$ \\
\hline$(\mathrm{RbCl})_{2}$ & 0.258 & $0.20[0.20]$ & 0.204 & $0.190^{\mathrm{f}}$ & $0.212^{\mathrm{f}}$ & & $0.188(22)$ \\
\hline$(\mathrm{RbBr})_{2}$ & 0.266 & $0.23[0.23]$ & & $0.201^{\mathrm{f}}$ & $0.224^{\mathrm{f}}$ & & $0.201(30)$ \\
\hline$(\mathrm{Rbl})_{2}$ & 0.274 & $0.24[0.23]$ & & $0.206^{\mathrm{f}}$ & $0.228^{\mathrm{f}}$ & & $0.249(70)$ \\
\hline$(\mathrm{CsF})_{2}$ & 0.221 & $0.22[0.20]$ & 0.097 & $0.227^{\mathrm{f}}$ & - & & $0.329(82)$ \\
\hline$(\mathrm{CsCl})_{2}$ & 0.260 & $0.23[0.26]$ & 0.224 & $0.212^{\mathrm{f}}$ & $0.278^{\mathrm{f}}$ & & $0.241^{\mathrm{e}}(62)$ \\
\hline$(\mathrm{CsBr})_{2}$ & 0.269 & $0.20[0.27]$ & & $0.222^{\mathrm{f}}$ & $0.241^{\mathrm{f}}$ & & $0.250(28)$ \\
\hline$(\mathrm{CsI})_{2}$ & 0.276 & $0.18[0.18]$ & 0.287 & $0.226^{\mathrm{f}}$ & $0.249^{f}$ & & 0.221 (166) \\
\hline
\end{tabular}

${ }^{\mathrm{a}}$ Reference 11 . Values in square brackets are calculated relative to experimental monomer data.

${ }^{\mathrm{b}}$ Reference 7 .

${ }^{\mathrm{c}}$ Reference 35 and this work (see Table III).

${ }^{\mathrm{d}}$ Reference 36.

${ }^{\mathrm{e}}$ Analyzed including multiple scattering.

${ }^{\mathrm{f}}$ Effective core calculations.

of $(\mathrm{LiF})_{2}$ only the first term of the Dunham expansion is used (harmonic potential) the bond expansion is reduced by $40 \%$ but the calculated bond angle increases only by $1.3^{\circ}$.

There are considerable differences in the predictions of the bond expansions from the different models (see Table VI). Let us first consider the Li compounds in some detail. Here the experimental bond expansions seem to be reliable and are in good agreement with ab initio calculations. Obviously the bond expansions from our model are much too long. Bond expansions from the shell model are very close to our values when they are calculated relative to shell model monomer bond lengths but are much smaller and in good agreement with experiment when they are calculated relative to experimental $r_{e}$ values. The DD model falls in between, still in reasonable agreement with experiment. This can be explained in the following way.

Our calculations start with the experimental monomer potential while the coefficients of the Born-Mayer repulsion term in the paper of Welch et al. have been derived from crystal data. When the bond length in the monomer is calculated with the shell model the result is much too small. ${ }^{11}$ While our model starts with the exact monomer bond length and gives a rather poor dimer bond length the shell model starting with crystal data gives fairly good dimer results but the monomer bond length is very poor. This is a strong indication that the repulsive potential changes from monomers to crystals and that a considerable part of this change shows up already between dimers and monomers, at least for the $\mathrm{Li}$ compounds. This is not too surprising because the monomer- dimer bond expansion is about $50 \%$ of the monomer-crystal expansion. In the DD model ${ }^{7(b)}$ calculations the changes of the repulsive potential have been taken into account by a rough interpolation between hardness parameters $\varrho_{M X}$ for monomers and $\varrho c_{M X}$ for crystals, resulting in data for the bond stretch which are between the results of Welch and ours.

The too long bond expansion resulting from our model can in no way be removed by improved effective polarizabilities and van der Waals or overlap repulsion terms between equally charged ions or by inclusion of higher order terms in the potential. Bond expansions and angles calculated with our program using fixed free ion polarizabilities instead of Seitz-Ruffa corrected values are in good agreement with the results given in Table II of Ref. 7(b) where a T-Rittner monomer model potential with a Born-Mayer repulsive term has been used. Therefore, it doesn't seem useful to persue a better analytic function with fixed constants to model the overlap repulsion between nearest neighbors. We have to conclude that these constants change when more than one nearest neighbor is present. This seriously limits the accuracy of predictions from ionic models.

Hence, the performance of the ionic models is somewhat disappointing for the dimer structures, given the presumably relatively simple binding in these systems. Since the ab initio results are in better agreement with experiment where comparisons can be made, one might suggest that one should use $a b$ initio methods on systems of this type and forego recourse to the simpler models. However, there are several 
good reasons for not adopting this approach. First, even in cases where $a b$ initio results are quite accurate, they do little to yield a simple picture of the bonding (beyond verifying that it is largely ionic). If quantum mechanics play a small role beyond determining the shape of the Pauli repulsive potential, one would expect to be able to model these interactions more accurately. In addition, while ab initio methods are quite simple to apply to the dimers, and, in fact, have been applied to significantly larger clusters, ${ }^{37,38}$ very large systems will likely be out of reach for the near future, and even when such calculations are tractable, general searches for the many local minima that exist will be a taxing computational problem. Thus it remains desirable to have a more accurate empirical model for use in modeling ionic systems. The results presented here clearly indicate, that the most important step in this direction would be a better understanding of the Pauling repulsion between the ions when more than one nearest neighbor is present.

\section{ACKNOWLEDGMENTS}

Financial support by the Deutsche Forschungsgemeinschaft under Sonderforschungsbereich 337 is gratefully acknowledged. R.J.C. gratefully acknowledges support from the National Science Foundation (Grant No. CHE-92228222) and the Camille and Henry Dreyfus Foundation for a Camille and Henry Dreyfus Teacher-Scholar Award (1993-1998). R.J.M. would like to thank the microwave group at the Freie Universität Berlin for their discussions and hospitality in several visits, as well as thank the donors of the Petroleum Research Fund, administrated by the American Chemical Society, for their support.

${ }^{1}$ M. Born and W. Heisenberg, Z. Phys. 23, 1 (1924).

${ }^{2}$ E. S. Rittner, J. Chem. Phys. 19, 1030 (1951).

${ }^{3}$ C. T. O'Konski and W. T. Higuchi, J. Chem. Phys. 23, 1175 (1955).

${ }^{4}$ T. A. Milne and D. Cubicciotti, J. Chem. Phys. 29, 846 (1958).

5 (a) J. Berkowitz, J. Chem. Phys. 29, 1386 (1958); (b) 32, 1519 (1960).

${ }^{6}$ G. M. Rothberg, J. Chem. Phys. 34, 2069 (1961).

${ }^{7}$ (a) P. Brumer and M. Karplus, J. Chem. Phys. 58, 3903 (1973); (b) 64, 5165 (1976).

${ }^{8}$ R. G. Gordon and Y. S. Kim, J. Chem. Phys. 56, 3122 (1972).

${ }^{9}$ H. Coker J. Phys. Chem. 80, 2078 (1976).

${ }^{10}$ S. Trugman and R. G. Gordon, J. Chem. Phys. 64, 4625 (1976).

${ }^{11}$ (a) D. O. Welch, O. W. Lazareth, G. Dines, and R. D. Hatcher, J. Chem. Phys. 64, 835 (1976); (b) 68, 2159 (1978).

${ }^{12}$ V. G. Solomonik and K. S. Krasnov, Zhur. Fiz. Khim. 53, 284 (1979) [Russ. J. Phys. Chem. 53, 161 (1979)].

${ }^{13}$ J. Shanker, H. B. Agrawal, and G. G. Agrawal, J. Chem. Phys. 73, 4056 (1980).

${ }^{14}$ J. Berkowitz, C. H. Batson, and G. L. Goodman, J. Chim. Phys. (Phys.Chim. Biol.) 77, 631 (1980).

${ }^{15}$ A. K. M. Islam, Can. J. Phys. 60, 865 (1982).

${ }^{16}$ J. Diefenbach and T. P. Martin, J. Chem. Phys. 85, 4585 (1985).

${ }^{17}$ B. T. Gowda, B. S. Sheriga, and S. W. Benson, J. Comp. Chem. 6, 93 (1985).

${ }^{18}$ B. I. Dunlap, J. Chem. Phys. 84, 5611 (1986).

${ }^{19}$ F. Ramondo, L. Bencivenni, and V. Rossi, J. Mol. Struct. 192, 73 (1989).

${ }^{20}$ L. A. Blomfield, C. W. S. Conover, Y. A. Yang, Y. J. Twu, and N. G. Phillips, Z. Phys. D 20, 93 (1991).
${ }^{21}$ N. G. Phillips, C. W. S. Conover, and L. A. Blomfield, J. Chem. Phys. 94, 4980 (1991).

${ }^{22}$ R. S. Chauhan, S. C. Sharma, S. B. Sharma, and B. S. Sharma, J. Chem. Phys. 95, 4397 (1991).

${ }^{23}$ J. M. Mestdagh and J. P. Visticot, Chem. Phys. 155, 79 (1991).

${ }^{24}$ (a) M. Kumar, A. J. Kaur, and J. Shanker, J. Chem. Phys. 84, 5735 (1986); (b) M. Kumar and J. Shanker, ibid. 96, 5289 (1992).

${ }^{25}$ J. P. Rose and R. S. Berry, J. Chem. Phys. 96, 517 (1992).

${ }^{26}$ R. L. Whetten, Acc. Chem. Res. 26, 49 (1993).

${ }^{27}$ M. Born and J. Mayer, Z Phys. 75, 1 (1932).

${ }^{28}$ L. Pauling, Proc. R. Soc. London, Ser. A 114, 181 (1927).

${ }^{29}$ R. J. Mawhorter, M. Fink, and J. G. Hartley, J. Chem. Phys. 83, 4418 (1985).

${ }^{30}$ J. G. Hartley and M. Fink, J. Chem. Phys. 87, 5477 (1987); 89, 6053 (1988); 89, 6058 (1988)

${ }^{31}$ S. B. Lapshina, G. V. Girichev, and A. Shlykov, Zhur. Strukt. Khim. 30, 49 (1989) [Russ. J. Struct. Chem. 30, 397 (1990)].

${ }^{32}$ S. H. Bauer, T. Ino and R. F. Porter, J. Chem. Phys. 33, 685 (1960).

${ }^{33}$ P. A. Akishin and N. G. Rambidi, Zh. Neorgan. Khim. 5, 23 (1960) [Russ. J. Inorg. Chem. 5, 10 (1960)]; Z. Phys Chem. (Leipzig) 213, 111 (1960).

${ }^{34}$ R. P. Dicke, D. Maurice, R. J. Cave, and R. J. Mawhorter, J. Chem. Phys. 98, 2182 (1993).

${ }^{35}$ R. J. Cave and I. Ono, J. Chem. Phys. 99, 9764 (1993).

${ }^{36}$ J. Modisette, L. Lou, and P. Nordlander, J. Chem. Phys. 101, 8903 (1994).

${ }^{37}$ C. Ochsenfeld and R. Ahlrichs, J. Chem. Phys. 97, 3487 (1992); Ber. Bunsenges. Phys. Chem. 96, 2287 (1992).

${ }^{38} \mathrm{P}$. Weis, C. Ochsenfeld, R. Ahlrichs, and M. M. Kappes, J. Chem. Phys. 97, 2553 (1992).

${ }^{39}$ B. R. Miller and L. S. Bartell, J. Chem. Phys. 72, 800 (1980).

${ }^{40}$ M. Blander, Alkali Halide Vapors (Academic, New York, 1979), p. 30.

${ }^{41}$ P. D. Kleinschmidt and K. M. Axler, High Temp. Sci. 28, 127 (1990).

${ }^{42}$ M. A. Williamson and P. D. Kleinschmidt, J. Nucl. Mater. 201, 115 (1993).

${ }^{43}$ P. G. Wahlbeck, D. L. Myers, T. L. Hendrixson, F. D. Pierson, and S. A. Pyles, J. Chem. Phys. 81, 915 (1992).

${ }^{44}$ J. Diefenbach and T. P. Martin, J. Chem. Phys. 83, 2238 (1985).

${ }^{45}$ J. L. Dunham, Phys. Rev. 41, 721 (1932).

${ }^{46}$ K. D. Jordan, J. L. Kinsey, and R. Silbey, J. Chem. Phys. 61, 911 (1974).

${ }^{47}$ H. W. DeWijn, J. Chem. Phys. 44, 810 (1966).

${ }^{48}$ F. Seitz, J. Chem. Phys. 6, 130 (1938).

${ }^{49}$ A. R. Ruffa, Phys. Rev. 130, 1412 (1963).

${ }^{50}$ E. W. Pearson, M. D. Jackson, and R. G. Gordon, J. Phys. Chem. 88, 119 (1984).

${ }^{51}$ D. L. Lacks and R. G. Gordon, Phys. Rev. B 48, 2889 (1993); J. Geophys. Res. B 98, 22, 147 (1993); Phys. Chem. Miner. 22, 145 (1995).

${ }^{52}$ A. L. Thakkar, J. Chem. Phys. 62, 1693 (1975).

${ }^{53}$ R. Narayan and S. Ramaseshan, J. Chem. Solids 37, 395 (1976); 39, 1287 (1978).

${ }^{54}$ J. A. Nelder and R. Mead, Comput. J. 7, 308 (1965).

${ }^{55}$ W. J. Hehre, R. Ritchfield, and J. A. Pople, J. Chem. Phys. 56, 2257 (1972); P. C. Hariharan and J. A. Pople, Theor. Chim. Acta 28, 213 (1973).

${ }^{56}$ W. R. Wadt and P. J. Hay, J. Chem. Phys. 82, 284 (1985).

${ }^{57}$ M. M. Hurley, L. F. Pacios, P. A. Christiansen, R. B. Ross, and W. C. Ermler, J. Chem. Phys. 84, 6840 (1986).

${ }^{58}$ L. A. LaJohn, P. A. Christiansen, R. B. Ross, T. Atashroo, and W. C. Ermler, J. Chem. Phys. 87, 2812 (1987).

${ }^{59}$ R. B. Ross, J. M. Powers, T. Atashroo, W. C. Ermler, L. A. LaJohn, and P. A. Christiansen, J. Chem. Phys. 93, 6654 (1990).

${ }^{60}$ M. J. Frisch, G. W. Trucks, M. Head-Gordon, P. M. Gill, M. W. Wong, J. B. Foresman, B. G. Johnson, H. B. Schlegel, M. A. Robb, E. S. Replogle, R. Gomperts, J. L. Andres, K. Raghavachari, J. S. Binkley, C. Gonzales, R. L. Martin, D. J. Fox, D. J. Defrees, J. Baker, J. J. P. Stewart, and J. A. Pople, Gaussian 92, Revision A, Gaussian, Inc., Pittsburgh, PA, 1992. 